\title{
The identification of novel targets of miR-16 and characterization of their biological functions in cancer cells
}

Xin Yan ${ }^{1+}$, Hongwei Liang ${ }^{2 \dagger}$, Ting Deng ${ }^{1 \dagger}$, Kegan Zhu $^{2}$, Suyang Zhang ${ }^{2}$, Nan Wang ${ }^{2}$ X Xueyuan Jiang ${ }^{2}$, Xueliang Wang ${ }^{2}$, Rui Liu' ${ }^{1}$, Ke Zen², Chen-Yu Zhang ${ }^{2^{*}}$, Yi Ba ${ }^{1^{*}}$ and Xi Chen ${ }^{2^{*}}$

\begin{abstract}
Background: In eukaryotes, miR-16 is an important microRNA (miRNA) that is involved in numerous biological processes. However, it is not fully understood how miR-16 executes its physiological functions. In the present study, we aimed to identify novel miR-16 targets and study their biological functions.

Methods: Candidate target genes of miR-16 were screened by microarray analysis of mRNA levels in several cancer cell lines with enhanced miR-16. Three bioinformatics algorithms, including TargetScan, PicTar, and miRanda, were used in combination to calculate the miR-16 targets. The expression levels of miR-16 and target mRNA were examined by relative quantification RT-PCR, and the expression levels of target protein were detected by Western blot. Luciferase reporter plasmids were constructed to confirm direct targeting. The effect of miR-16 and target gene on cell viability was evaluated using MTT assays. The effects of miR-16 and target gene on apoptosis and cell cycle distribution were evaluated by flow cytometry analysis.

Results: By overexpressing miR-16 in several cancer cell lines and measuring global mRNA levels using microarray analysis, we identified 27 genes that may be regulated by miR-16. After the bioinformatics filtering process, 18 genes were selected as candidate miR-16 targets. Furthermore, we experimentally validated three of these candidates, MAP7 (microtubule-associated protein 7), PRDM4 (PR domain containing 4) and CDS2 (CDP-diacylglycerol synthase 2), as direct targets of miR-16. Finally, we demonstrated that miR-16 targeting MAP7 played a critical role in regulating proliferation but not apoptosis and cell cycle progression in cancer cells.
\end{abstract}

Conclusion: In summary, the present study identifies several novel miR-16 targets and illustrates a novel function of miR-16 targeting MAP7 in modulating proliferation in cancer cells.

Keywords: microRNA, miR-16, MAP7, PRDM4, CDS2

\section{Introduction}

microRNAs (miRNAs) are a class of endogenous noncoding RNAs, typically 22 nucleotides in length, that function primarily by targeting the 3 '-untranslated region ( $3^{\prime}$-UTR) of specific mRNAs and hence silence gene expression by either translational repression or

\footnotetext{
* Correspondence: cyzhang@nju.edu.cn; yiba99@yahoo.com; xichen@nju.edu.cn ${ }^{\dagger}$ Equal contributors

2Jiangsu Engineering Research Center for microRNA Biology and

Biotechnology, State Key Laboratory of Pharmaceutical Biotechnology, School of Life Sciences, Nanjing University, 22 Hankou Road, Nanjing 210093, China

'Tianjin Medical University Cancer Institute and Hospital, Huanhuxi Road, Tiyuanbei, Tianjin 300060, China
}

direct mRNA degradation [1,2]. Through these posttranscriptional gene regulation mechanisms, miRNAs regulate a wide range of biological processes, including cell proliferation and differentiation, migration, apoptosis, development and metabolism [1,2].

The number of miRNAs encoded by the genomes of different organisms varies dramatically, and more than 2000 miRNAs have been identified in humans [1,2]. Some of these miRNAs have attracted special attention for their involvement in the initiation, progression and metastasis of human cancers [3-5]. One particularly well-studied example is the ubiquitously expressed and highly conserved miR-16, one of the first miRNAs to be

\section{Biomed Central}

(c) 2013 Yan et al.; licensee BioMed Central Ltd. This is an Open Access article distributed under the terms of the Creative Commons Attribution License (http://creativecommons.org/licenses/by/2.0), which permits unrestricted use, distribution, and reproduction in any medium, provided the original work is properly cited. 
linked to human malignancies [6]. Evidence indicates that miR-16 can modulate the cell cycle, inhibit cell proliferation, promote cell apoptosis and suppress tumorigenicity both in vitro and in vivo [7]. These effects can be explained by several targets of miR-16: the antiapoptotic gene Bcl-2 (B-cell lymphoma 2) [8]; numerous genes involved in the G1-S transition, such as cyclin D1, cyclin D3, cyclin E1 and CDK6 (cyclin-dependent kinase 6) [9-11]; and genes involved in the Wnt signaling pathway, such as WNT3A (wingless-type MMTV integration site family, member 3A) [11]. Consistently, miR-16 is frequently deleted and/or downregulated in many types of cancer, such as chronic lymphocytic leukemia [6,12], prostate cancer [11] and lung cancer [13].

Given the importance of miR-16 during tumorigenesis, the aim of the present study is to identify new miR-16 targets and study their biological functions in cancer cells. By overexpressing miR-16 in several cancer cell lines and measuring global mRNA levels through microarray platforms, we identified a large number of transcripts that are potentially regulated by miR-16. We further confirmed MAP7 (microtubule-associated protein 7), PRDM4 (PR domain containing 4) and CDS2 (CDP-diacylglycerol synthase 2) as direct targets of miR-16. The mechanism through which miR-16 executes its functions in cancer cells was also investigated in this study.

\section{Methods \\ Cells}

The human lung adenocarcinoma cell line A549, human breast cancer cell line MCF-7, human epithelial carcinoma cell line HeLa, human colon adenocarcinoma cell line SW480 and human embryonic kidney cell line HEK-293 were purchased from the Shanghai Institute of Cell Biology, Chinese Academy of Sciences (Shanghai, China). These cells were maintained in RPMI 1640 medium (Gibco, CA, USA) or DMEM medium (Gibco) supplemented with $10 \%$ fetal bovine serum (FBS) (Gibco). Cells were grown at $37^{\circ} \mathrm{C}$ in a humidified atmosphere with $5 \% \mathrm{CO}_{2}$.

\section{miR-16 overexpression or knockdown}

miR-16 overexpression was achieved by transfecting cells with pre-miR-16 (a synthetic RNA oligonucleotide duplex mimicking miR-16 precursor), while miR-16 knockdown was achieved by transfecting cells with anti-miR-16 (a chemically modified single-stranded antisense oligonucleotide designed to specifically target against mature miR-16). Scrambled negative control RNA (pre-miR-control and anti-miR-control) served as negative control. Synthetic RNA molecules, including premiR-16, anti-miR-16 and scrambled negative control RNA, were purchased from GenePharma (Shanghai,
China). Cells were seeded on $60-\mathrm{mm}$ dishes and were transfected the following day using Lipofectamine 2000 (Invitrogen, Carlsbad, CA, USA) according to the manufacturer's instructions. For each well, equal doses (200 pmol) of pre-miR-control, pre-miR-16, anti-miR-control or anti-miR-16 were added. Cells were harvested $24 \mathrm{~h}$ after transfection.

\section{RNA isolation and relative quantification RT-PCR}

Total RNA was extracted from the cultured cells using TRIzol Reagent (Invitrogen) according to the manufacturer's instructions. Assays to quantify mature miR16 were carried out using Taqman microRNA probes (Applied Biosystems, Foster City, CA, USA) according to the manufacturer's instructions, with slight modification. Briefly, $1 \mu \mathrm{g}$ of total RNA was reverse-transcribed to cDNA using AMV reverse transcriptase (TaKaRa, Dalian, China) and a stem-loop RT primer (Applied Biosystems). The reaction conditions were: $16^{\circ} \mathrm{C}$ for $30 \mathrm{~min}, 42^{\circ} \mathrm{C}$ for $30 \mathrm{~min}, 85^{\circ} \mathrm{C}$ for $5 \mathrm{~min}$. Real-time PCR was performed using a TaqMan PCR kit on an Applied Biosystems 7300 Sequence Detection System (Applied Biosystems). The reactions were incubated in a 96-well optical plate at $95^{\circ} \mathrm{C}$ for $10 \mathrm{~min}$, followed by 40 cycles of $95^{\circ} \mathrm{C}$ for $15 \mathrm{~s}$ and $60^{\circ} \mathrm{C}$ for $1 \mathrm{~min}$. All reactions were run in triplicate. After the reactions, the threshold cycles $\left(C_{T}\right)$ values were determined using fixed threshold settings, and the mean $C_{T}$ was determined from the triplicate PCRs. In the experiments presented here, a comparative $C_{T}$ method was used to compare each condition with controls. miRNA expression in cells was normalized to that of the U6 snRNA. The amount of miR-16 relative to the internal control U6 was calculated with the equation $2^{-\Delta \Delta C T}$, in which $\Delta \Delta \mathrm{C}_{\mathrm{T}}=\left(\mathrm{C}_{\mathrm{T} \text { miR }-16}-\mathrm{C}_{\mathrm{T}} \text { U6 }\right)_{\text {target }}-\left(\mathrm{C}_{\mathrm{T} \text { miR }-16-}\right.$ $\left.\mathrm{C}_{\mathrm{T} \text { U6) }}\right)_{\text {control. }}$.

For relative quantification RT-PCR analysis of MAP7, PRDM4 and $\beta$-actin mRNA, $1 \mu \mathrm{g}$ of total RNA was reverse-transcribed to cDNA with oligo dT and Thermoscript (TaKaRa, Dalian, China) in the reaction conditions: $42^{\circ} \mathrm{C}$ for $60 \mathrm{~min}$ and $70^{\circ} \mathrm{C}$ for $10 \mathrm{~min}$. Then real-time PCR was performed on an Applied Biosystems 7300 Sequence Detection System (Applied Biosystems) using SYBR green dye (Invitrogen). The 20- $\mu$ l PCR reaction included $1 \mu \mathrm{l}$ RT product, $1 \times$ QuantiTect SYBR green PCR Master Mix, and $0.5 \mu \mathrm{M}$ each sense and antisense primers. The reactions were incubated in a 96-well plate at $95^{\circ} \mathrm{C}$ for $5 \mathrm{~min}$, followed by 40 cycles of $95^{\circ} \mathrm{C}$ for $30 \mathrm{~s}, 60^{\circ} \mathrm{C}$ for $30 \mathrm{~s}$, and $72^{\circ} \mathrm{C}$ for $30 \mathrm{~s}$. All reactions were run in triplicate. After the reactions, the $\mathrm{C}_{\mathrm{T}}$ values were determined using fixed threshold settings. The relative amount of MAP7 and PRDM4 mRNA was normalized to $\beta$-actin mRNA. The sequences of the primers are as follows: MAP7 (sense): 5'-AAACTCTTTGTAACACC ACCTGA-3'; MAP7 (antisense): 5' -GATGGAGATACA 
GCCCTTCG-3'; PRDM4 (sense): 5'-CGAAAGATTCA TGGTGGAAA-3'; PRDM4 (antisense): 5' -TAAGGTGG TGGAGGTAGGGT- $3^{\prime} ; \beta$-actin (sense): 5 '-AGGGAAAT CGTGCGTGAC-3'; and $\beta$-actin (antisense): $5^{\prime}$-CGCTC ATTGCCGATAGTG-3'.

\section{Microarray procedures}

The commercially available $22 \mathrm{~K}$ Human Genome Array was purchased from the CapitalBio Corporation (Beijing, China). Labeling, hybridization, washing, and scanning were performed according to the standard operating procedure provided by CapitalBio. Briefly, total RNA was used to synthesize cDNA in an in vitro transcription reaction. cDNA was fluorescently labeled by $\mathrm{Cy} 5$ or Cy3-CPT using the Klenow enzyme. After hybridization, non-specifically bound molecules were removed from the microarray with two consecutive washes $0.2 \%$ SDS and $2 \times \mathrm{SSC}$ at $42^{\circ} \mathrm{C}$ for 5 minutes followed by $0.2 \%$ SSC for 5 minutes at room temperature). Subsequently, the arrays were scanned with a LuxScan 10KA confocal laser scanner (CapitalBio Corporation), and the obtained images were analyzed using LuxScan Version 3.0 (CapitalBio Corporation) employing the LOWESS normalization method.

\section{miR-16 target prediction}

The miRNA target prediction and analysis was performed with the algorithms from TargetScan (http://www.targetscan. org/) PicTar (http://pictar.mdc-berlin.de/) and miRanda (http://www.microrna.org/).

\section{Western blotting}

MAP7 and PRDM4 protein levels were quantified by western blot analysis of whole cell extracts using antibodies against MAP7 and PRDM4. These samples were normalized by blotting with an antibody against $\alpha$-tubulin. Anti-MAP7 (NBP1-46240) antibody was purchased from Novus (CO, USA), and anti-PRDM4 (sc-15254) and anti- $\alpha$-tubulin (B-7) antibodies were purchased from Santa Cruz Biotechnology (CA, USA).

\section{Luciferase assay}

The entire 3'-UTRs of human CDS2, PRDM4, MAP7, PPP1R11, CHUK, LAMP2 and SLC35A4 were amplified from human genomic DNA using PCR. The PCR products were inserted into the p-MIR-report plasmid (Ambion). Efficient insertion was confirmed by sequencing. For luciferase reporters containing mutant CDS2, PRDM4 and MAP7 3'-UTRs, the sequences that interact with bases $2-8$ of the miR-16 seed sequence were mutated. For luciferase reporter assays, cells were cultured in 6-well plates, and each well was transfected with $2 \mu \mathrm{g}$ of firefly luciferase reporter plasmid, $2 \mu \mathrm{g}$ of $\beta$-galactosidase expression plasmid (Ambion), and equal amounts of scrambled negative control RNA, pre-miR-16, or anti-miR-16 using Lipofectamine 2000 (Invitrogen). The $\beta$-galactosidase plasmid was used as a transfection control. At $24 \mathrm{~h}$ post-transfection, cells were assayed using luciferase assay kits (Promega, Madison, WI, USA). The data depicted are representative of three independent experiments performed on different days.

\section{Plasmid construction and siRNA interference assay}

A mammalian expression plasmid encoding the human MAP7 open reading frame (pReceiver-M02-MAP7) was purchased from GeneCopoeia (Germantown, MD, USA). An empty plasmid served as a negative control. The siRNA (sequence: CAGAUUAGAUGUCACCAAUTT) targeting human MAP7 cDNA was designed and synthesized by Invitrogen (Carlsbad, CA, USA). A scrambled siRNA (Stealth ${ }^{\text {Ta }}$ RNAi negative control kit, Invitrogen, Carlsbad, CA, USA) that could not target human MAP7 cDNA was included as a negative control. Plasmid and siRNA were transfected into A549 cells using Lipofectamine 2000 (Invitrogen) according to the manufacturer's instructions. Total RNA and protein was isolated at $24 \mathrm{~h}$ post-transfection. The MAP7 mRNA and protein expression levels were assessed by relative quantification RT-PCR and western blotting.

\section{Cell viability assay}

A549 cells were plated at $2.5 \times 10^{3}$ cells per well in 96-well plates and incubated overnight in DMEM medium supplemented with 10\% FBS. After transfection, $20 \mu \mathrm{l} 3$ (4,5-dimethylthiazol-2-yl)-2,5-diphenyl tetrazolium bromide (MTT) $(5 \mathrm{mg} / \mathrm{mL})$ was added into a corresponding test well and incubated for $4 \mathrm{~h}$. The supernatant was then discarded, and $200 \mu \mathrm{L}$ of DMSO was added to each well to dissolve the precipitate. Optical density (OD) was measured at a wavelength of $570 \mathrm{~nm}$.

\section{Apoptosis assays}

Apoptosis was detected using an Annexin V-FITC/ propidium iodide (PI) staining assay. A549 cells were cultured in 12-well plates and transfected with $40 \mathrm{pmol}$ of pre-miR-16 or siRNA of MAP7 to induce apoptosis. Pre-miR-control and control siRNA served as negative controls. Cells were cultured overnight with both serumcontaining complete medium and serum-depleted medium; the attached cells and floating cells were then harvested. Flow cytometry analysis of apoptotic cells was carried out using an Annexin V-FITC/PI staining kit (BD Biosciences, CA, USA). After washes with cold PBS, the cells were resuspended in binding buffer $(100 \mathrm{mM}$ HEPES, pH 7.4, $100 \mathrm{mM} \mathrm{NaCl}$, and $25 \mathrm{mM} \mathrm{CaCl}_{2}$ ) followed by staining with Annexin V-FITC/PI at room temperature in darkness for $15 \mathrm{~min}$. Apoptotic cells were then evaluated by gating PI and Annexin V- 
positive cells on a fluorescence-activated cell-sorting (FACS) flow cytometer (BD Biosciences, San Jose, CA). All experiments were performed in triplicate.

\section{Cell cycle assay}

Cells were harvested, washed once with PBS, and fixed in $70 \%$ ethanol overnight. Staining for DNA content was performed with $50 \mathrm{mg} / \mathrm{ml}$ propidium iodide and $1 \mathrm{mg} / \mathrm{ml}$ RNase A for $30 \mathrm{~min}$. Analysis was performed on a FACS flow cytometer (BD Biosciences) with Cell Quest Pro software. Cell cycle modeling was performed with Modfit 3.0 software (Verity Software House, Topsham, ME).

\section{Statistical analysis}

All presented images of western blotting and cell cycle assay are representative of at least three independent experiments. Relative quantification RT-PCR, luciferase reporter, and cell viability assays were performed in triplicate, and each experiment was repeated three to five times. The data shown are the mean \pm SD of at least three independent experiments. Statistical significance was considered at $\mathrm{p}<0.05$ using the Student's t-test.

\section{Results}

The identification of candidate miR-16 targets by microarray analysis and bioinformatics algorithms

Animal miRNAs were originally believed to block translational processes without affecting transcript levels [1-3]. However, recent evidence has changed this view, showing that target mRNA degradation is a widespread effect of miRNA-based regulation that alone accounts for most of the repression mediated by miRNAs in mammalian cell cultures [14]. Inspired by this mode of miRNA action, we postulated that the high-throughput mRNA microarray assays capable of detecting such effects at the mRNA level would provide a promising avenue for miRNA target identification. Considering that miR-16 is a ubiquitously expressed miRNA frequently downregulated in many types of cancer, we chose several cell lines with very different origins for microarray analysis to identify the common targets of miR-16. The selected human cell lines included A549 (lung adenocarcinoma cell), MCF-7 (breast cancer cell), HeLa (epithelial carcinoma cell), SW480 (colon adenocarcinoma cell) and HEK-293 (embryonic kidney cell). We transfected A549, MCF-7, HeLa, SW480 and HEK-293 cells with equal doses of pre-miR-16 (miRNA mimic) or pre-miR-control (scrambled miRNA mimic, as a negative control) and then surveyed mRNA transcripts that were inversely expressed relative to miR-16 using microarray analysis. The results showed that although most mRNAs were not influenced by transfection with miR-16, some mRNAs were downregulated in pre-miR-16-transfected cells compared with control cells (Figure 1). To reduce the false positive rate and obtain a more accurate assessment of the genuine miR-16 targets, only the mRNAs that were downregulated by a factor of at least 1.5 (0.66-fold downregulation) in at least four cell lines were considered as putative miR-16 targets. Finally, 27 mRNAs were selected for further analysis (Table 1).

Next, three algorithms, including TargetScan [15], PicTar [16], and miRanda [17], were used in combination to calculate whether the downregulated mRNAs were candidate miR-16 targets. Only the mRNAs predicted as miR-16 targets by at least two of the above-mentioned algorithms were considered positive. In total, 18 mRNAs were identified as candidate miR-16 targets (Table 2).

\section{Identification of direct miR-16 targets by luciferase reporter screening}

However, transcript-based expression analysis and computational predictions alone cannot directly measure the actual miRNA-target interaction. Therefore, a luciferase reporter assay was conducted to screen for target genes that were directly controlled by miR-16 through the 3 'UTR. Three genes, including MAP7, PRDM4 and CDS2, were selected. The predicted interaction between miR16 and these genes is illustrated in Figure 2A. The entire 3 '-UTRs of these genes were fused into a downstream position of the firefly luciferase gene in a reporter plasmid. Luciferase reporters containing the 3'-UTRs of PPP1R11 (protein phosphatase 1 regulatory subunit 11), CHUK (conserved helix-loop-helix ubiquitous kinase), LAMP2 (lysosomal-associated membrane protein 2) and SLC35A4 (solute carrier family 35, member A4) were generated and served as negative controls (PPP1R11, LAMP2 and SLC35A4 contain putative miR-16 binding sites but were not significantly altered by transfection with miR-16, while CHUK does not have a candidate miR-16 binding sequence). The resulting plasmids were transfected into A549 cells combined with pre-miR-16 or pre-miR-control. The luciferase activities were assayed $36 \mathrm{~h}$ after transfection. As shown in Figure 2B, overexpression of miR-16 significantly decreased the luciferase activities of the reporter containing the 3 '-UTRs of MAP7, PRDM4 and CDS2, whereas the negative control reporters were unaffected by miR-16 in A549 cells.

Furthermore, we introduced point mutations into the corresponding seed complementary sites in the 3'-UTR of MAP7, PRDM4 and CDS2 to eliminate the predicted binding by miR-16 (Figure 2A). Luciferase reporters containing either wild-type or mutant MAP7, PRDM4 and CDS2 3'-UTRs were co-transfected with pre-miR-16, pre-miR-control, anti-miR-16 (miRNA antisense) or antimiR-control (scrambled miRNA antisense as negative control) into A549 cells. While the overexpression of miR16 decreased the luciferase activity, the inhibition of miR16 resulted in a significant increase in the luciferase 


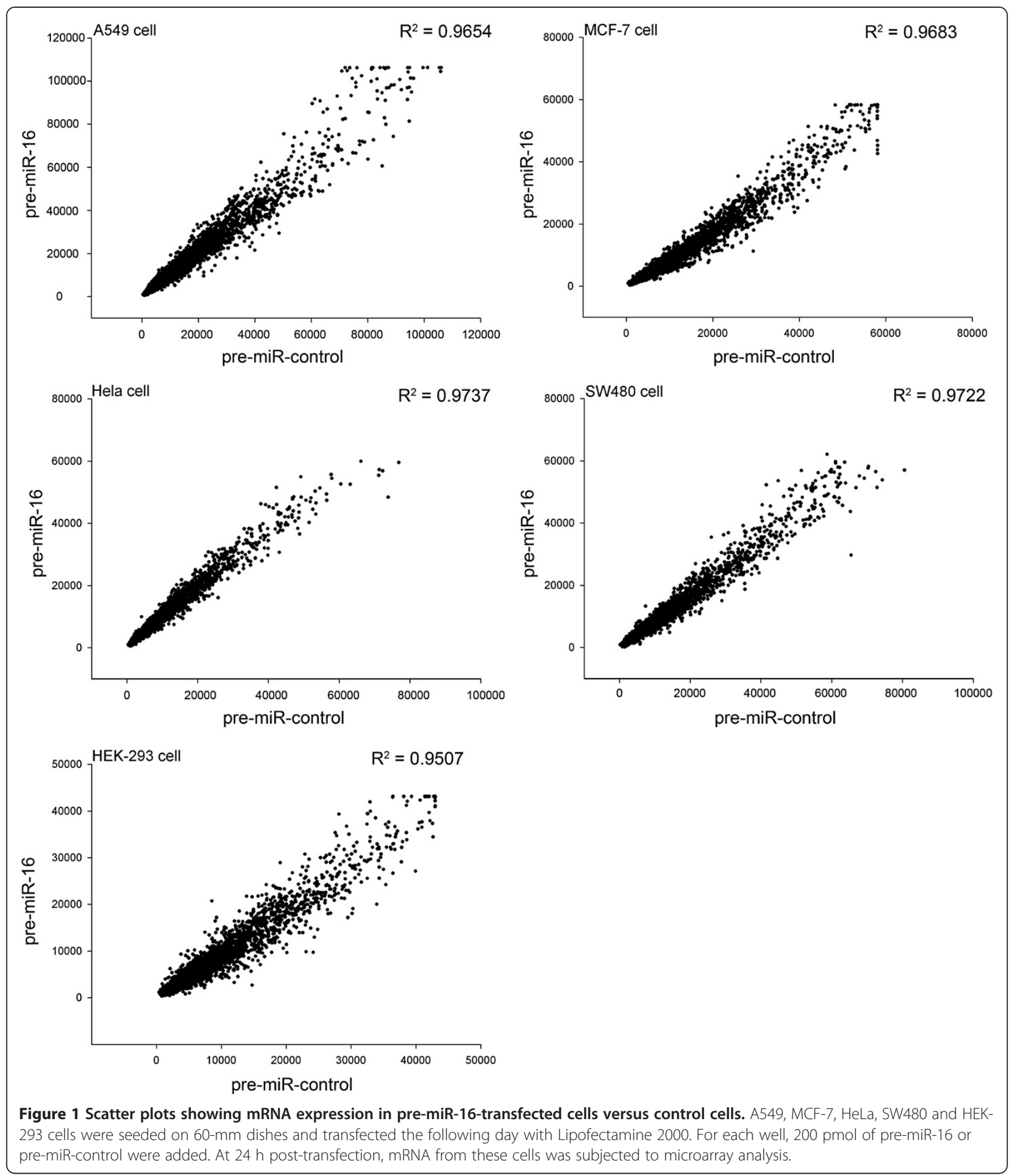

activity, and mutations in the seed complementary sites almost fully rescued the repression of reporter activity by miR-16 (Figure 2C). These results demonstrate that miR16 can directly recognize the 3 '-UTRs of MAP7, PRDM4 and CDS2 and mediate the post-transcriptional inhibition of these genes.
The downregulation of MAP7 and PRDM4 expression by miR-16

We then determined whether the overexpression or knockdown of miR-16 had an impact on MAP7 and PRDM4 expression. We transfected A549, HeLa and MCF-7 cells with equal doses of pre-miR-16, pre-miR-control, anti-miR-16 or 
Table 1 mRNAs identified as downregulated in cells overexpressing miR-16

\begin{tabular}{ccccccc}
\hline Gene name & A549 & MCF-7 & Hela & SW480 & HEK-293 & Mean fold \\
\hline ARL2 & 0.4438 & 0.3506 & & 0.5400 & 0.1817 & 0.3790 \\
\hline CDS2 & 0.5127 & 0.3022 & 0.5133 & & 0.3045 & 0.4082 \\
\hline ANAPC13 & & 0.3843 & 0.6239 & 0.3393 & 0.4607 & 0.4520 \\
\hline NUPL1 & & 0.3315 & 0.6230 & 0.5340 & 0.3525 & 0.4602 \\
\hline MAP7 & 0.6168 & 0.4428 & 0.6220 & 0.5151 & 0.2027 & 0.4799 \\
\hline ARG2 & 0.4384 & 0.5793 & & 0.4731 & 0.4845 & 0.4938 \\
\hline RTN4 & 0.6063 & 0.4024 & & 0.5277 & 0.4415 & 0.4945 \\
\hline RARS & 0.6351 & 0.4953 & 0.5922 & & 0.2686 & 0.4978 \\
\hline PRDM4 & 0.6458 & 0.3892 & 0.5288 & 0.3809 & 0.5978 & 0.5085 \\
\hline SPRYD3 & 0.6232 & 0.3945 & 0.6387 & 0.5158 & 0.3778 & 0.5100 \\
\hline ATF6 & 0.5601 & 0.5654 & & 0.5141 & 0.4159 & 0.5139 \\
\hline TMEM109 & & 0.5647 & 0.5863 & 0.4650 & 0.4396 & 0.5139 \\
\hline RPS6KA3 & 0.5621 & 0.4471 & 0.6515 & 0.5522 & 0.3611 & 0.5148 \\
\hline BCR & & 0.6217 & 0.5980 & 0.4826 & 0.3719 & 0.5185 \\
\hline KIF3B & 0.6569 & 0.5500 & 0.5970 & 0.3850 & 0.4665 & 0.5311 \\
\hline ENTPD6 & 0.5493 & 0.5145 & 0.5929 & 0.4446 & 0.5748 & 0.5352 \\
\hline CCND3 & & 0.4777 & 0.5767 & 0.4867 & 0.6042 & 0.5363 \\
\hline ARHGDIA & & 0.5038 & 0.6295 & 0.5048 & 0.5103 & 0.5371 \\
\hline GABARAPL1 & & 0.5492 & 0.6459 & 0.6228 & 0.3693 & 0.5468 \\
\hline PLEKHB2 & & 0.4670 & 0.6040 & 0.5178 & 0.6149 & 0.5509 \\
\hline FL11149 & & 0.4721 & 0.6577 & 0.6297 & 0.5081 & 0.5669 \\
\hline DNAJC5 & & 0.5794 & 0.6497 & 0.4857 & 0.5730 & 0.5720 \\
\hline ZNF622 & & 0.6543 & 0.6059 & 0.6394 & 0.4159 & 0.5789 \\
\hline ANXA11 & 0.6548 & 0.5811 & & 0.4592 & 0.6410 & 0.5840 \\
\hline VPS33B & 0.6586 & 0.6122 & & 0.5394 & 0.5359 & 0.5865 \\
\hline ANLN & & 0.4597 & 0.6433 & 0.6247 & 0.6247 & 0.5881 \\
\hline PTH2 & 0.5264 & 0.6352 & & 0.5819 & 0.6600 & 0.6009 \\
\hline & & & & & & \\
\hline
\end{tabular}

anti-miR-control, and analyzed the expression levels of MAP7 mRNA by relative quantification RT-PCR at $24 \mathrm{~h}$ post-transfection. The expression of miR-16 was abolished by the introduction of anti-miR-16, whereas pre-miR-16 significantly increased miR-16 levels in A549, HeLa and MCF-7 cells (Figure 3, A, D and G). Cells transfected with pre-miR-16 showed reduced levels of MAP7 mRNA relative to cells transfected with pre-miR-control; in contrast, the expression levels of MAP7 mRNA were significantly increased in anti-miR-16transfected cells compared with those transfected with anti-miR-control (Figure 3, B, E and H). Furthermore, we repeated the above experiments and determined the expression of MAP7 protein by western blotting at $24 \mathrm{~h}$ post-transfection. The expression levels of the MAP7 protein were significantly abolished by the introduction of pre-miR-16, whereas cells transfected with pre-miR-control maintained a considerable amount of MAP7 protein; in contrast, anti-miR-16 significantly increased the expression levels of MAP7 protein in A549, HeLa and MCF-7
Table 2 Candidate miR-16 targets predicted by TargetScan, PicTar and miRanda

\begin{tabular}{|c|c|c|c|c|}
\hline Gene name & TargetScan & PicTar & miRanda & Result \\
\hline ARL2 & 4 & 5 & 1 & Positive \\
\hline CDS2 & 2 & 2 & 1 & Positive \\
\hline ANAPC13 & 1 & 0 & 0 & \\
\hline NUPL1 & 0 & 0 & 0 & \\
\hline MAP7 & 2 & 2 & 2 & Positive \\
\hline ARG2 & 0 & 0 & 0 & \\
\hline RTN4 & 1 & 0 & 1 & Positive \\
\hline RARS & 0 & 0 & 0 & \\
\hline PRDM4 & 1 & 1 & 1 & Positive \\
\hline SPRYD3 & 4 & 0 & 2 & Positive \\
\hline ATF6 & 1 & 0 & 1 & Positive \\
\hline TMEM109 & 2 & 0 & 2 & Positive \\
\hline RPS6KA3 & 2 & 0 & 1 & Positive \\
\hline$B C R$ & 1 & 1 & 1 & Positive \\
\hline KIF3B & 1 & 0 & 1 & Positive \\
\hline ENTPD6 & 2 & 0 & 0 & \\
\hline CCND3 & 2 & 0 & 1 & Positive \\
\hline ARHGDIA & 4 & 5 & 1 & Positive \\
\hline GABARAPL1 & 3 & 0 & 2 & Positive \\
\hline PLEKHB2 & 2 & 0 & 0 & \\
\hline FLJ11149 & 0 & 0 & 0 & \\
\hline DNAJC5 & 2 & 0 & 0 & \\
\hline ZNF622 & 1 & 1 & 1 & Positive \\
\hline ANXA11 & 1 & 0 & 1 & Positive \\
\hline VPS33B & 2 & 0 & 1 & Positive \\
\hline ANLN & 1 & 0 & 1 & Positive \\
\hline PTH2 & 0 & 0 & 0 & \\
\hline
\end{tabular}

Numbers in the table indicate the putative binding sites of miR-16 in the 3'-UTR of target genes.

cells (Figure 3, C, F and I). Moreover, we found that the PRDM4 mRNA and protein levels were inversely correlated to miR-16 in A549 cells (Additional file 1: Figure S1, $A$ and $B)$. These results demonstrate that miR-16 regulates the expression of MAP7 and PRDM4 at both the transcript and protein levels.

\section{The role of miR-16 in regulating MAP7 during tumorigenesis}

We next focused on studying the role of miR-16 in regulating MAP7 and PRDM4. Because miR-16 is known to be involved in regulation of proliferation, apoptosis and cell cycle progression, we investigated whether the overexpression or knockdown of miR-16, MAP7 or PRDM4 would have an impact on these cellular phenotypes in A549 cells. To knock down MAP7 and PRDM4, siRNAs against MAP7 or PRDM4 were transfected into A549 cells. To overexpress MAP7 and PRDM4, recombinant plasmids designed to specially express the full- 


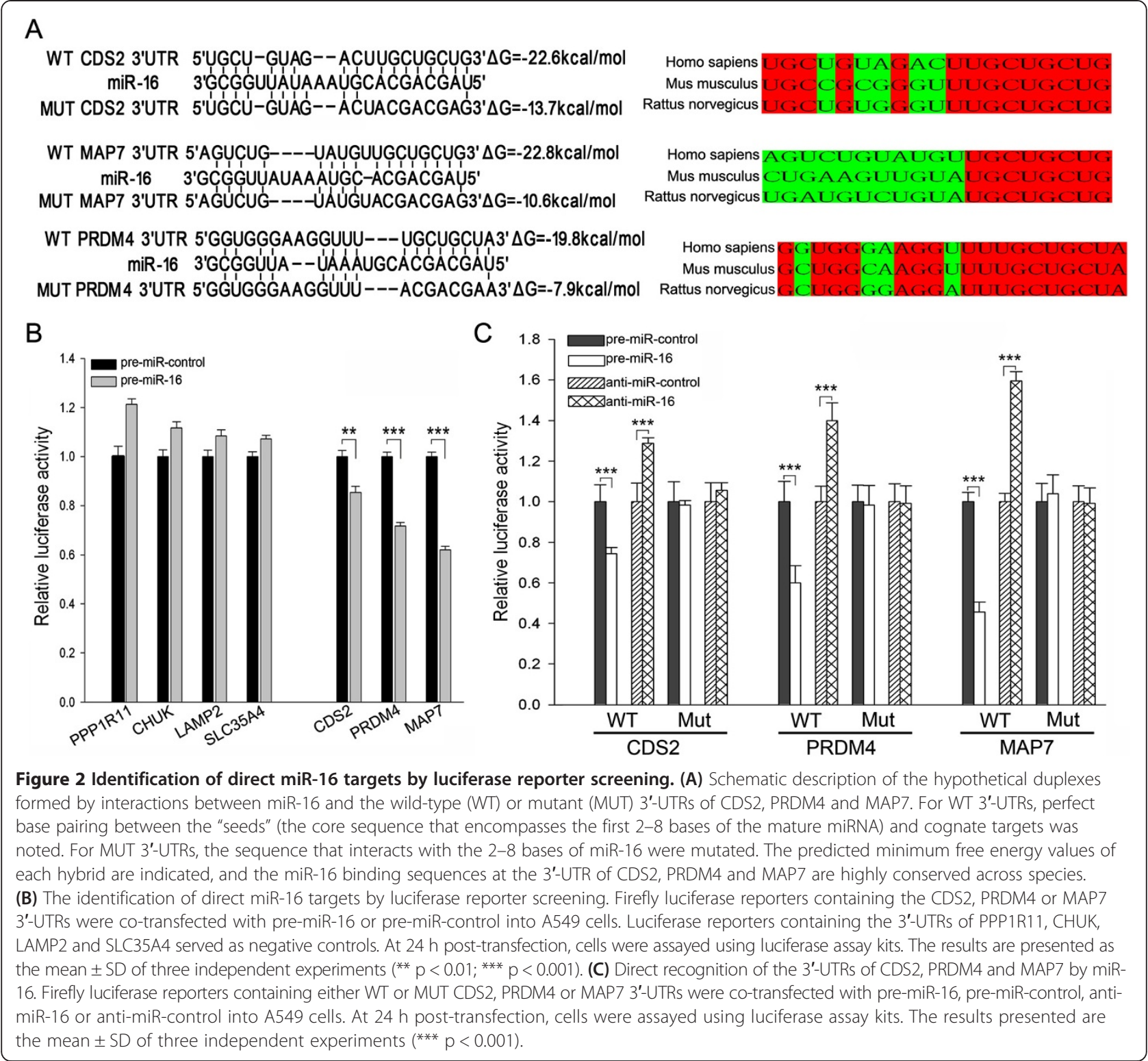

length open reading frame of MAP7 and PRDM4 without the miR-16-responsive 3'-UTRs were constructed and transfected into A549 cells. The efficient overexpression or knockdown of MAP7 (Figure 4, A and B) and PRDM4 (Additional file 1: Figure S1, C and D) is shown.

In supporting the notion that miR-16 is essential in repressing proliferation, A549 cells transfected with antimiR-16 showed stimulation of cell proliferation; in contrast, overexpression of miR-16 had an opposite effect on cell proliferation (Figure 4C). Furthermore, we assessed the role of MAP7 and PRDM4 on cell proliferation. A549 cells transfected with a MAP7 overexpressing plasmid proliferated at a significantly higher rate, whereas the knockdown of MAP7 by siRNA significantly reduced proliferation (Figure 4D). Finally, compared to cells transfected with pre-miR-16, cells transfected with premiR-16 and MAP7 overexpressing plasmid exhibited significantly higher proliferation rates (Figure 4E), suggesting that miR-16-resistant MAP7 could rescue the suppression of MAP7 by miR-16. These results demonstrate that miR-16 can inhibit cell proliferation by silencing MAP7. On the other hand, no differences were observed in proliferation rates between the cells transfected with control siRNA or siRNA against PRDM4 (Additional file 1: Figure S1E). The results suggest that miR-16 could modulate cell proliferation by downregulating genes other than PRDM4.

Next, we investigated apoptosis in cells with enhanced miR-16 or silenced MAP7 or PRDM4 by flow cytometry analysis. Statistically significantly more apoptotic cells 


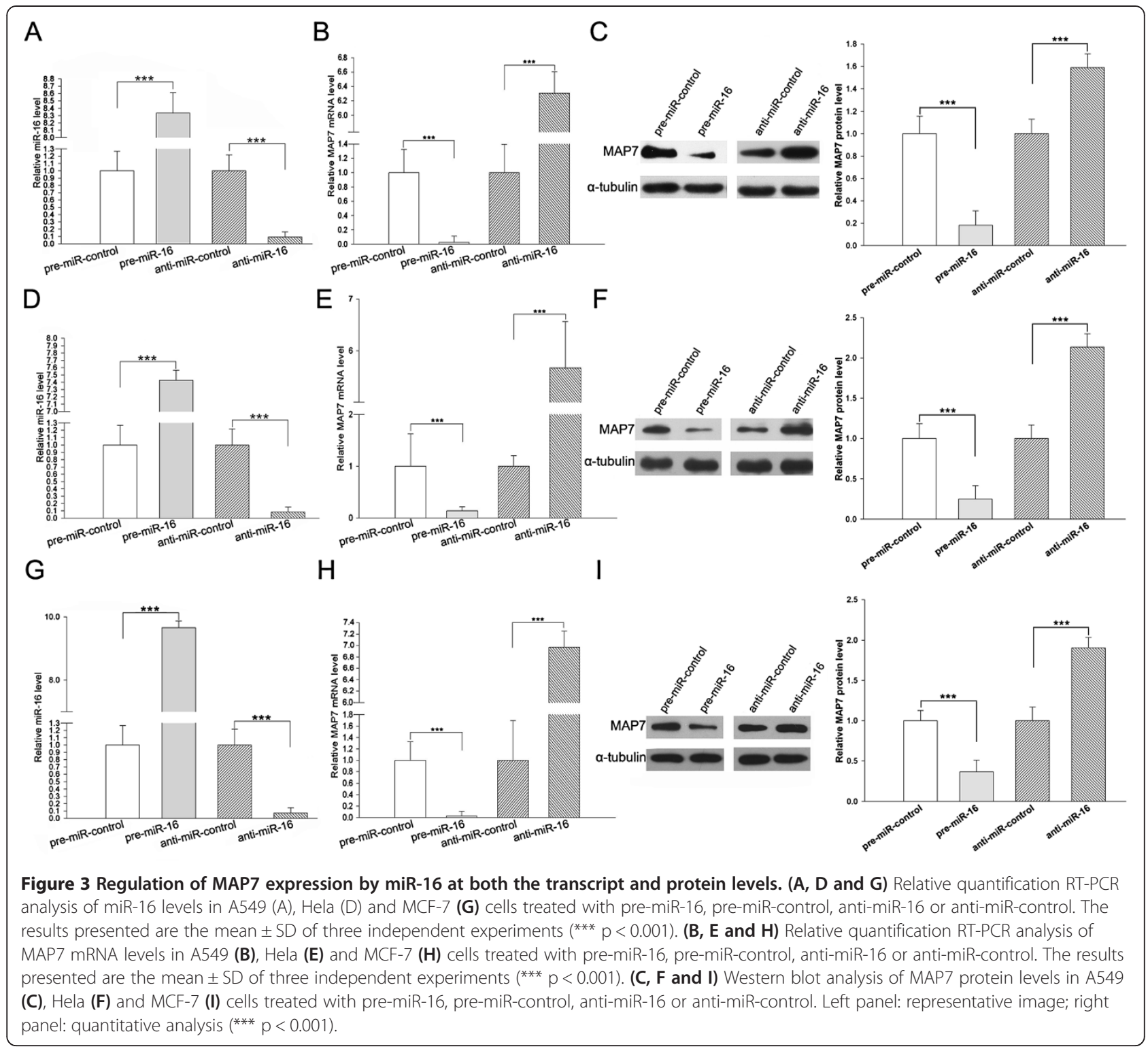

were observed in the pre-miR-16-transfected cells compared with pre-miR-control-transfected cells (Figure 4, $F$ and G). However, no differences in the levels of apoptosis were observed in cells transfected with negative control siRNA or siRNA against MAP7 (Figure 4, F and G). Likewise, silencing of PRDM4 had no effect on apoptosis in A549 cells (Additional file 1: Figure S1, F and G). These results suggest that miR-16 could trigger apoptosis by downregulating genes other than MAP7 and PRDM4.

Finally, we investigated cell cycle distribution in cells with enhanced miR-16 or silenced MAP7 or PRDM4 by flow cytometry analysis. Compared with cells transfected with pre-miR-control, cells transfected with pre-miR-16 triggered an accumulation of cells in the G0/G1 stage, whereas the numbers of cells in the $S$ and $G 2 / M$ phases decreased (Figure 4, H and I). Transfection with siRNA against MAP7 partially yielded the phenotype generated by overexpression of miR-16, but the effects were not significant, featured by a lower G0/G1 cell accumulation but a higher $\mathrm{S}$ and G2/M cell accumulation (Figure 4, H and I). Moreover, silencing of PRDM4 had no effect on cell cycle progression of A549 cells (Additional file 1: Figure S1, $\mathrm{H}$ and I). The results suggest that miR-16 might negatively regulate cell cycle progression from the G0/G1 phase to the $\mathrm{S}$ phase by silencing genes other than MAP7 and PRDM4.

\section{Discussion}

Although the number of known miRNAs is continuously increasing, information regarding their precise cellular function remains limited. One of the major challenges in 


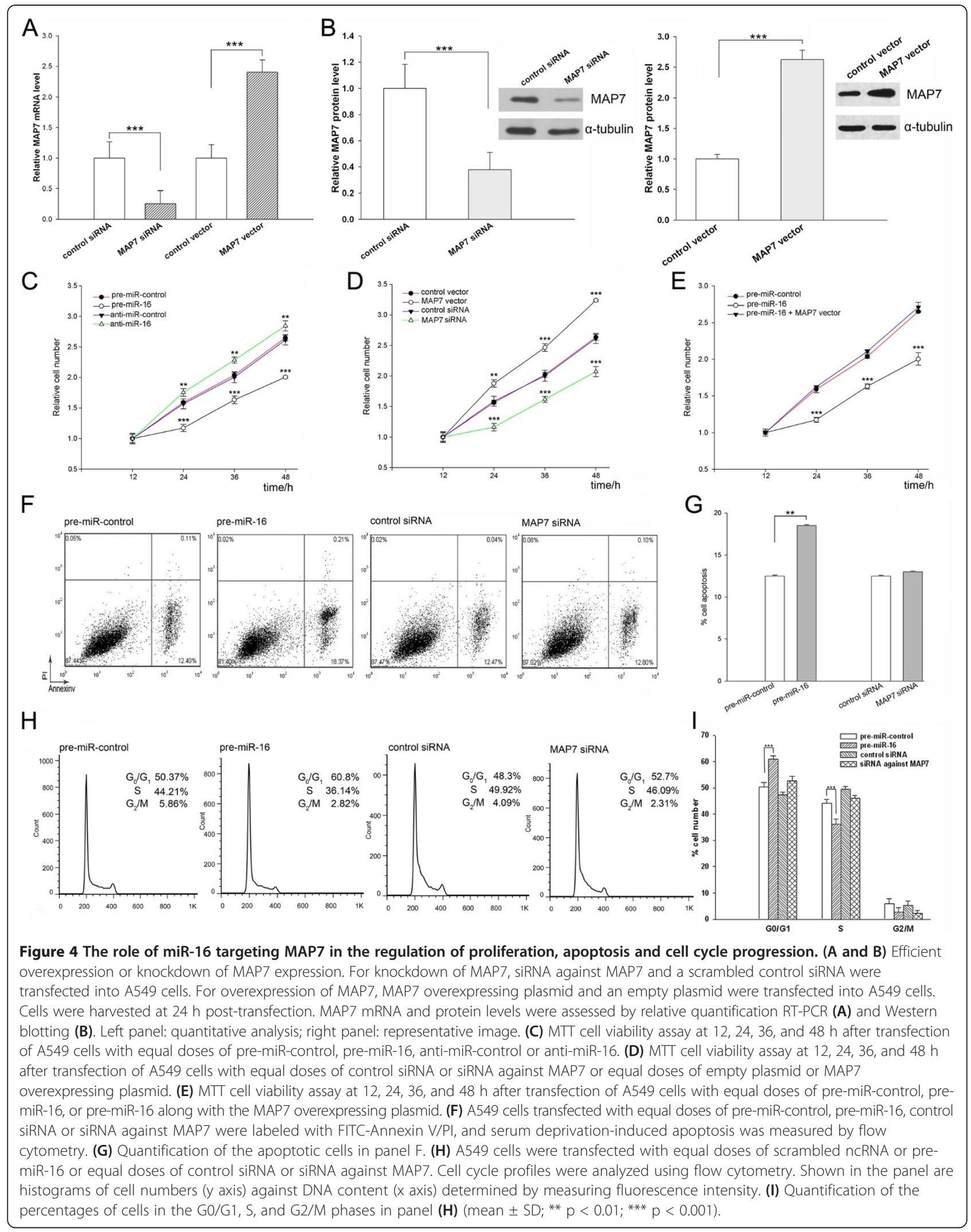


understanding the functions of a specific miRNA is to identify its genuine target genes. To date, only a few miRNAs have been assigned target mRNAs. In the present study, we aimed to identify the genuine targets of miR-16, a miRNA that has long been thought to be implicated in tumorigenesis. By using microarray analysis to globally screen the expression patterns of transcripts in several cell lines with overexpressed miR-16 and by combining bioinformatics programs to select genuine miR-16 targets from the differentially regulated genes, we identified a panel of 18 candidate miR-16 target genes. Actually, the same strategy has been used in our earlier study [18]. In that study, in order to identify the common targets of miR-16, we performed the microarray analysis in A549, MCF-7 and HEK-293 cells with either enhanced or silenced miR-16 [18]. In this study, we re-performed the microarray analysis in A549, MCF-7 and HEK-293 cells (we have re-performed the whole procedure of the microarray analysis, from preparation of new mRNA samples to analysis of new data), as well as in additional HeLa and SW480 cells with enhanced miR-16. There are some overlap of microarray data between this study and our earlier study. Among the 27 mRNAs identified as downregulated in cells overexpressing miR-16, nine (ARL2, CDS2, ANAPC13, MAP7, ARG2, RTN4, RARS, SPRYD3 and RPS6KA3) were also selected by our earlier study. The consistency between these two studies further demonstrates the robustness of our strategy to identify the common miR-16 targets. On the other hand, the additional 18 mRNAs identified as downregulated in this study were not observed in the prior analysis. This may be due to the different criteria we used to select miR-16 targets. In our previous study, only the mRNAs that showed consistent downregulation (fold change $<0.66$ ) in all three cell lines were considered as candidate miR-16 targets. In the present study, the mRNAs that showed downregulation (fold change $<0.66$ ) in at least four cell lines were considered as putative miR-16 targets. Thus, for mRNAs such as NUPL1, TMEM109 and CCND3, because they did not show significant downregulation in A549 cells, they were not included as candidate miR-16 targets in our previous study. However, in the present study they were included because they showed downregulation in other four cell lines. We have provided a table to summarize the comparison findings between these two studies (Additional file 1: Table S1).

Subsequently, we randomly selected three genes, MAP7, PRDM4 and CDS2, from the candidate 18 genes and used a luciferase reporter screen to experimentally demonstrate that all three of these genes are directly targeted by miR-16. Indeed, we demonstrated that miR16 regulates the expression of MAP7 and PRDM4 at both the transcript and protein levels through several biological approaches. The results demonstrate that our strategy of combining global gene expression analysis with bioinformatics prediction may provide good selection criteria to aid in miRNA target identification.

We also showed that miR-16 could regulate cell proliferation but not apoptosis and cell cycle progression in A549 cells by silencing MAP7. However, how MAP7 regulates cell proliferation is currently unknown. MAP7 is a microtubule-associated protein that is predominantly expressed in cells of epithelial origin [19]. Microtubule-associated proteins are involved in microtubule dynamics, which are essential for many important cellular processes including cell division, motility and differentiation [19]. MAP7 has been shown to modulate microtubule functions [19]. Thus, MAP7 may function as a proliferation promoter through its role in the microtubule dynamics. Furthermore, the role of MAP7 in cancer progression is also unclear. In support of a potential role for MAP7 in metastatic growth, this gene was recently identified as one out of only fifteen that was highly upregulated in metastatic endometrial cancer using a 22 K Affymetrix array [20]. Moreover, high MAP7 expression has been associated with tumor recurrence and poor prognosis in Stage II colon cancer patients [21]. Here, we showed that MAP7 had the potential to accelerate proliferation, but not influence apoptosis and cell cycle progression in A549 cells. Although we did not investigate the consequence of miR16 targeting MAP7 in other cancer cell lines, as miR-16 could regulate the expression of MAP7 in A549, HeLa and MCF-7 cells, we speculate that miR-16 might have similar cellular functions through silencing MAP7. Therefore, the downregulation of miR-16 in cancer cells would, in theory, relieve the suppression of miR-16 on MAP7, which in turn accelerates tumorigenesis. The modulation of MAP7 protein level by miR-16 may explain, at least in part, why the downregulation of miR-16 can promote cancer progression. Additional studies are necessary to fully elucidate the exact roles of miR-16 in regulating MAP7 in other cancer cells. Additional studies are necessary to uncover the clinical effects of miR16 in regulating MAP7 during cancer progression.

In this study, we uncovered multiple targets of miR-16 by combining mRNA microarray profiles with bioinformatics analysis. We further experimentally validated MAP7 as a direct target of miR-16 and demonstrated that this targeting plays a critical role in regulating proliferation in cancer cells.

\section{Additional file}

Additional file 1: Figure S1. Regulation of PRDM4 expression by miR16. (A) Relative quantification RT-PCR analysis of PRDM4 mRNA levels in A549 cells treated with pre-miR-16, pre-miR-control, anti-miR-16 or anti- 
miR-control. (B) Western blot analysis of PRDM4 protein levels in A549 cells treated with pre-miR-16, pre-miR-control, anti-miR-16 or anti-miRcontrol. Left panel: representative image; right panel: quantitative analysis. (C and D) Efficient overexpression or knockdown of PRDM4 expression. For knockdown of PRDM4, siRNA against PRDM4 and a scrambled control siRNA were transfected into A549 cells. For overexpression of PRDM4, PRDM4 overexpressing plasmid and an empty plasmid were transfected into A549 cells. Cells were harvested at $24 \mathrm{~h}$ post-transfection. PRDM4 mRNA and protein levels were assessed by relative quantification RT-PCR (C) and Western blotting (D). Left panel: quantitative analysis; right panel: representative image. (E) MTT cell viability assay at 12, 24, 36, and 48 h after transfection of A549 cells with equal doses of control siRNA or siRNA against PRDM4. (F) A549 cells transfected with equal doses of premiR-control, pre-miR-16, control siRNA or siRNA against PRDM4 were labeled with FITC-Annexin V/PI, and serum deprivation-induced apoptosis was measured by flow cytometry. (G) Quantification of the apoptotic cells in panel $F$. (H) A549 cells were transfected with equal doses of control siRNA or siRNA against PRDM4. Cell cycle profiles were analyzed using flow cytometry. Shown in the panel are histograms of cell numbers (y axis) against DNA content ( $x$ axis) determined by measuring fluorescence intensity. (I) Quantification of the percentages of cells in the G0/G1, S, and G2/M phases in panel H. (mean \pm SD; ${ }^{* *} \mathrm{p}<0.01 ;{ }^{* *} \mathrm{p}<0.001$ ). Table S1. mRNAs identified as downregulated in cells overexpressing miR-16 by this study and our previous study.

\section{Competing interests}

The authors declare that they have no competing interests.

\section{Authors' contributions}

$X C, Y B$ and $C Y Z$ designed research and analyzed data. XY, HL, TD, KZ, SZ, NW and $X J$ performed experiments. XW and RL performed statistical analysis. KZ made contributions to the conception and design of experiments. $X C$ and $Y B$ wrote the manuscript. All authors read and approved the final manuscript.

\section{Acknowledgements}

This work was supported by grants from the National Natural Science Foundation of China (Nos. 81101330, 81201946, 31271378 and 81250044) and the Natural Science Foundation of Jiangsu Province (Nos. BK2011013 and BK2012014). This work was also supported by the program for New Century Excellent Talents in University from the Ministry of Education, China (NCET-09-0898 and NCET-12-0261).

\section{Author details}

'Tianjin Medical University Cancer Institute and Hospital, Huanhuxi Road, Tiyuanbei, Tianjin 300060, China. ${ }^{2}$ Jiangsu Engineering Research Center for microRNA Biology and Biotechnology, State Key Laboratory of Pharmaceutical Biotechnology, School of Life Sciences, Nanjing University, 22 Hankou Road, Nanjing 210093, China.

Received: 7 February 2013 Accepted: 13 August 2013

Published: 14 August 2013

\section{References}

1. Pasquinelli AE: MicroRNAs and their targets: recognition, regulation and an emerging reciprocal relationship. Nat Rev Genet 2012, 13:271-282.

2. Peláez N, Carthew RW: Biological robustness and the role of microRNAs: a network perspective. Curr Top Dev Biol 2012, 99:237-255.

3. Nana-Sinkam SP, Croce CM: Clinical applications for microRNAs in cancer. Clin Pharmacol Ther 2013, 93:98-104.

4. Melo SA, Kalluri R: Molecular pathways: microRNAs as cancer therapeutics. Clin Cancer Res 2012, 18:4234-4239.

5. Lages E, Ipas H, Guttin A, Nesr H, Berger F, Issartel JP: MicroRNAs: molecular features and role in cancer. Front Biosci 2012, 17:2508-2540.

6. Calin GA, Dumitru CD, Shimizu M, Bichi R, Zupo S, Noch E, Aldler H, Rattan S, Keating M, Rai K, Rassenti L, Kipps T, Negrini M, Bullrich F, Croce CM: Frequent deletions and down-regulation of micro- RNA genes miR15 and miR16 at 13q14 in chronic lymphocytic leukemia. Proc Natl Acad Sci USA 2002, 99:15524-15529.
7. Aqeilan Rl, Calin GA, Croce CM: miR-15a and miR-16-1 in cancer: discovery, function and future perspectives. Cell Death Differ 2010 17:215-220

8. Cimmino A, Calin GA, Fabbri M, lorio MV, Ferracin M, Shimizu M, Wojcik SE, Aqeilan RI, Zupo S, Dono M, Rassenti L, Alder H, Volinia S, Liu CG, Kipps TJ, Negrini M, Croce CM: miR-15 and miR-16 induce apoptosis by targeting BCL2. Proc Natl Acad Sci USA 2005, 102:13944-13949.

9. Linsley PS, Schelter J, Burchard J, Kibukawa M, Martin MM, Bartz SR, Johnson JM, Cummins JM, Raymond CK, Dai H, Chau N, Cleary M, Jackson AL, Carleton M, Lim L: Transcripts targeted by the microRNA-16 family cooperatively regulate cell cycle progression. Mol Cell Biol 2007 , 27:2240-2252.

10. Liu Q, Fu H, Sun F, Zhang H, Tie Y, Zhu J, Xing R, Sun Z, Zheng X: miR-16 family induces cell cycle arrest by regulating multiple cell cycle genes. Nucleic Acids Res 2008, 36:5391-5404.

11. Bonci D, Coppola V, Musumeci M, Addario A, Giuffrida R, Memeo L, D'Urso L, Pagliuca A, Biffoni M, Labbaye C, Bartucci M, Muto G, Peschle C, De Maria R: The miR-15a-miR-16-1 cluster controls prostate cancer by targeting multiple oncogenic activities. Nat Med 2008, 14:1271-1277.

12. Calin GA, Ferracin M, Cimmino A, Di Leva G, Shimizu M, Wojcik SE, lorio MV, Visone R, Sever NI, Fabbri M, Iuliano R, Palumbo T, Pichiorri F, Roldo C, Garzon R, Sevignani C, Rassenti L, Alder H, Volinia S, Liu CG, Kipps TJ, Negrini M, Croce CM: A MicroRNA signature associated with prognosis and progression in chronic lymphocytic leukemia. N Engl J Med 2005, 353:1793-1801.

13. Bandi N, Zbinden S, Gugger M, Arnold M, Kocher V, Hasan L, Kappeler A, Brunner T, Vassella E: miR-15a and miR-16 are implicated in cell cycle regulation in a $\mathrm{Rb}$-dependent manner and are frequently deleted or down-regulated in non-small cell lung cancer. Cancer Res 2009, 69:5553-5559.

14. Huntzinger $E$, Izaurralde $E$ : Gene silencing by microRNAs: contributions of translational repression and mRNA decay. Nat Rev Genet 2011, 12:99-110.

15. Lewis BP, Shih IH, Jones-Rhoades MW, Bartel DP, Burge CB: Prediction of mammalian microRNA targets. Cell 2003, 115:787-798.

16. Krek A, Grün D, Poy MN, Wolf R, Rosenberg L, Epstein EJ, MacMenamin P, da Piedade I, Gunsalus KC, Stoffel M, Rajewsky N: Combinatorial microRNA target predictions. Nat Genet 2005, 37:495-500.

17. John B, Enright AJ, Aravin A, Tuschl T, Sander C, Marks DS: Human MicroRNA targets. PLoS Biol 2004, 2:e363.

18. Wang K, Li P, Dong Y, Cai X, Hou D, Guo J, Yin Y, Zhang Y, Li J, Liang H, Yu $B$, Chen J, Zen K, Zhang J, Zhang CY, Chen X: A microarray-based approach identifies ADP ribosylation factor-like protein 2 as a target of microRNA-16. J Biol Chem 2011, 286:9468-9476.

19. Masson D, Kreis TE: Binding of E-MAP-115 to microtubules is regulated by cell cycle-dependent phosphorylation. J Cell Biol 1995, 131:1015-1024.

20. Ferguson SE, Olshen AB, Viale A, Awtrey CS, Barakat RR, Boyd J: Gene expression profiling of tamoxifen-associated uterine cancers: evidence for two molecular classes of endometrial carcinoma. Gynecol Oncol 2004 92:719-725.

21. Blum C, Graham A, Yousefzadeh M, Shrout J, Benjamin K, Krishna M, Hoda R, Hoda R, Cole DJ, Garrett-Mayer E, Reed C, Wallace M, Mitas M: The expression ratio of Map7/B2M is prognostic for survival in patients with stage II colon cancer. Int J Oncol 2008, 33:579-584.

doi:10.1186/1476-4598-12-92

Cite this article as: Yan et al: The identification of novel targets of miR-

16 and characterization of their biological functions in cancer cells. Molecular Cancer 2013 12:92. 